\title{
Research on Information Models and Modelling Methods of the New Generation of OSS for Data Management
}

\author{
Y. Lin, Y.B. Wang \\ School of Information Engineering \\ Minzu University of China \\ China
}

\author{
Y.B. Wang, H. Zhang, J.H. Yang \\ Institute for Network Science \& Cyberspace \\ Tsinghua University \\ China
}

\begin{abstract}
In order to build the information model of the new generation of Operation Support System (OSS), we firstly point out that OSS is currently entering the era of big data management, and the Telco Operators are now facing big challenges. We then summarize the difficulties to build the OSS unified information model. After that, the paper surveys the existing information models, and modelling methodologies for OSS, and analyzes their applicability, advantages, as well as disadvantages. Finally, the idea of building the OSS unified information model is put forward. Moreover, the modelling idea has been applied in practice.
\end{abstract}

Keywords-data management; OSS; information model; modelling method

\section{INTRODUCTION}

In the past few years, when a new business or technology arose, the telecom operators traditionally added a new system to meet the new business needs. In the short term, operators can quickly introduce new services in a short time. But in the long term, this may lead to hundreds or even thousands different systems in the OSS [1]. Moreover, in the initial stage of building OSS systems, due to limitations of the planning and technology theory, all the systems exist independently. So, it is difficult to share information between systems. Facing the "silo problem"[2, 3] network management systems, telecom operators have to take the way of "swivel chair"[4] to manage the telecommunication networks. The way of "swivel chair" is not only costly and inefficient, but also difficult to make full use of OSS operation data. Therefore, telecom operators need to integrate the existing systems to break the "information silos".

\section{THE DIFFICULTIES OF BUILDING THE OSS UNIFIED INFORMATION MODEL}

Nowadays, information and communication technology is developing and changing. The internet of things, cloud computing, big data and other new technologies are promoting the development of enterprises, but also bring new challenges to OSS. Cloud computing is obviously here to stay, and deploying OSS to cloud computing is also inevitable. In the cloud computing environment, in order to ensure the OSS can take its advantages fully, changing the architecture of the OSS is considered. In the big-data era, a large number of semistructured and unstructured data swarm into OSS. How to use existing infrastructure to store these data and fully mine the value of OSS operation data is the problem that must be considered by the new generation of OSS. Therefore, OSS has entered the era of data management, and data has become the key factor of business management. Building the OSS unified information model is a challenging job. Below are some main difficulties:

1. The problem of systems integration: In the initial stage of building OSS systems, due to limitations of the planning and technology theory, each subsystem of OSS has its own independent information model. Homonym or synonym exists generally. In addition, due to historical reasons, the information produced by these multi-level subsystems is heterogeneous, which become the obstacle to share information between systems. So, how to integrate existing systems, break "information silos" and re-build each system model to form the OSS unified information model is a problem.

2. The expansibility of model: The world is constantly changing and developing, and building OSS is also a ongoing process. Many new subsystems will be added to the OSS in the future. So, the information model must has certain flexibility and expansibility. That means, without extra change, the model can be used directly in the future.

3. The diversity of data sources: Take the comprehensive analysis system of China Mobile for example, it has 44 data sources in total, and the systems are built independently. Homonym or synonym exists in these systems. It is difficult to ensure the consistency and availability of data and control the quality of the data.

4. How to change the structure of OSS: Faced with the challenges of big data, The Internet of things, cloud computing and other new technologies, telecom operators must consider changing the OSS's architecture. For example, in order to adapt the flexibility of Cloud computing scheduling, existing systems need to be decoupled effectively; the scheme of combining Structured Query Language (SQL)/Not Only Structured Query Language (NoSQL) and On-Line Transaction Processing (OLTP)/On-Line Analytical Processing (OLAP) needs to be adapted to store structured and unstructured data. 
Therefore, building the OSS unified information model is not easy, not only needs to consider the current situation, integrating the existing systems to share information, but also needs to make the model expandable in the future.

\section{RESEARCH ON INFORMATION MODELS}

For years, several management information models have been proposed by international organizations, which have greatly promoted the development of theoretical research on telecom network management information model. These international organizations include International Organization for Standardization (ISO), Internet Engineering Task Force (IETF), Telecommunication Management Forum (TMF), 3rd Generation Partnership Project (3GPP), 3rd Generation Partnership Project 2 (3GPP2), Distributed Management Task Force (DMTF), and International Telecommunication Union (ITU) and so on. According to different applications, they can be divided into three major categories, as shown in Table 1.

Simple Network Management Protocol (SNMP), which is a kind of network management protocols, is widely used in TCP/IP network. MIB [5] defines a collection which consists of managed objects. However, MIB does not well show the relationships between managed objects. A number of MIBs are proposed by the IETF, but only for certain products. And abstract and reusable concepts and structures are not defined. CIM [6] is the information model adopted by Web-Based Enterprise Management (WBEM) framework. It is an information model used to describe the computing and operating entities in enterprise network, rather than for telecom network management. Therefore, CIM needs to be made the necessary extensions for telecom management. But a unified extension mechanism is lacking, which may make extended information models based on CIM have incompatible problems.

TABLE I. THE CLASSIFICATION OF INTERNATIONAL ORGANIZATIONS.

\begin{tabular}{|c|c|c|}
\hline categories & organizations & models \\
\hline Internet management & IETF & $\begin{array}{c}\text { Management Information } \\
\text { Base (MIB) }\end{array}$ \\
\hline IT management & DMTF & $\begin{array}{c}\text { Common Information } \\
\text { Model (CIM) }\end{array}$ \\
\hline \multirow{5}{*}{ Telecommunication } & ITU & Generic Network \\
Information Model \\
\end{tabular}

According to Table 1 and the above description, GNIM [7], IF [8], CaSMIM [9], MTNM [10], IRP[11] and MIB(ISO)[12] have a certain referential value to model the new generation of OSS for data management.

IRP, CaSMIM, MTNM and GNIM are interface-oriented management information. Among them, IRP, CaSMIM and MTNM are top-down modelling methods, and the modelling process is basically the same: analyzing requirements, then defining the relevant model, and finally giving the solutions. IRP defines the Northbound Interface between Network Element Management Layer (NEML) and Network Management Layer (NML) in 3rd Generation. CaSMIM models the interface between the network management layer and service management layer. This service interface can be used to connect network management system and service management system. CaSMIM is a service management information model, which is one of its advantages. The managed objects in CaSMIM come from the M3100 and G805. However, the consistency of information between CaSMIM and ITU information model can't be guaranteed. MTNM models the interface between the NEML and NML, and it is a generic model used on multi-technology network. One of the MTNM's advantages is that it describes a management information model for the multi-technology network. However, it does not propose a service management model, and does not define the relationship with CaSMIM. The focus of GNIM is to model the physical resources in Telecommunications Management Network (TMN), and GNIM takes Guidelines for the Definition of Managed Objects (GDMO) [13] and Abstract Syntax Notation One (ASN.1) as its modelling language. The concept of object is used by GNIM to describe Network Element (NE). This allows managers to abstract network and service components to design a general model. However, bottom-up design method is used by TMN, which makes the TMN just pay attention to network element management, not model the system from the perspective of the whole network.

Information Framework is based on service model defined by enhanced Telecom Operations Map (eTOM) Level 0. In addition, Information Framework abstracts and analyzes all kinds of business information involved in the business processes, then defines various Aggregation Business Entities and Business Entities and divides them into different management domains in order to maximize information and data sharing. However, Information Framework is a generic model framework, and not just for a particular telecom operators. So, Information Framework has redundancy in practice. Moreover, it lacks data analysis. Information Framework only plays a reference role in practice.

So, Most of the information models focus on ordinary network management, can't fully meet the requirements of the new OSS network management. Furthermore, most research on models focus on theoretical level, while only part describes the modelling process, which do not embody typical design ideas for modelling the new generation of OSS.

\section{RESEARCH ON INFORMATION MODELLING METHODS}

Currently, the modelling methods proposed by international organizations include UTRAD (United TMN Requirements, Analysis and Design) [14], IRP and SANRR[15]. UTRAD proposed by ITU uses object-oriented 
analysis and design principle, and details three the stages of defining interface specification, which is traceable and iterative. The process of defining TMN interface specification is divided into requirement phase, analysis phase and design phase, and how to model interface by using object-oriented technology is given. IRP proposed by 3GPP divides the modelling process into requirement analysis phase, information service phase and solutions phase. SANRR proposed by TMF is a methodology that describes New Generation Operations System and Software (NGOSS) lifecycle. It is iterative, and includes five steps: scope, analyze, normalize, rationalize and rectify.

UTRAD and IRP are very similar; they are all interface modelling methods. SANRR is not a new method, but is the product that integrates all kinds of knowledge in the software field. SANRR reflects software engineering thinking. It is imperfect and immature. Further, since the modelling process described by SANRR is not detailed, it is difficult to guide the telecom operators to model OSS in practice.

Building OSS unified information model is an important and challenging job. So, it is a research hotpot. At present, the studies mainly focus on two aspects: one is how to solve the interoperability between existing models, another is the extensibility of the model. According to the above description, there are several management information models, but they are different on the classification and description of managed objects. How to integrate these information models is the key to solve the problem of interoperability between models. At present, the usual practice is to adopt a new technology to rephrase or re-build a higher level unified information model on the basis of these models. In order to solve the interoperability between management information models, [16, 17] introduced the ontology theory into the network management. [18] did not re-build a new unified information model to cover current existing information models, instead of using Semantic Web and Linked Data technology to describe various information models. [19] adopted Unified Modelling Language (UML) and associated the additional components with others through semantic relationships.

Currently, Shared Information Data/Model (SID) has been widely accepted by telecom operators. OSS is divided into different management function areas by SID. And it offers a common information model framework for building the actual OSS system. According to the data sources, functions and business, network data are divided into 3 dimensions by [20], and based on the SID, data are divided into customer domain, business domain, network domain and public resource domain. The unified modelling process includes data abstraction, model representation and relationship mining. But its modelling process is too simple to model in practice. Reference [21] takes eTOM as the reference model to define the business processes in detail and takes SID as the basic model to define and extend the information model. The basic idea of building the OSS unified information model is to map the business processes into eTOM, and then map the business entities of eTOM to SID. However, the business processes of each telecom operator may not be consistent with eTOM, it is difficult to map the business processes to eTOM.

\section{CONCLUSION}

Building the information model is the basis and premise of developing OSS system. Building the OSS unified information model can unify and standardize the system data, maximize the value of data and improve the quality of data. The traditional "silo problem" OSS system has been a serious obstacle to information sharing. So telecom operators must integrate the existing systems to break the "information silos".

However, the existing information model standards lag behind the development of telecom network, and can't meet the demands. In addition, the existing information models and modelling method are not general. So, a new OSS management information model needs to be built to meet the data management needs. To build the OSS unified information model, the following ideas are put forward:

1. pay attention to the existing information models. The existing information models in telecom domain should be valued when building the OSS unified information model. For example, IF is a generic information model framework, which analyzes the core business processes of telecom enterprises and compartmentalizes different function areas. IF is a generic information model, which make it need to be reconstructed according to the actual situation.

2. Learn from mainstream modelling methods, and combine top-down with bottom-up modelling method. Although the existing modelling methods have some limitations, they describe the key steps in modelling process. For example, the modelling process of SANRR includes five steps, although each step is not detailed. Therefore, every step needs to be detailed in practice. The specific modelling process should include the following steps: scope, analyze, normalize, rationalize, verify and rectify.

3. Use design patterns. Design patterns are the solution to representative problems in certain circumstance. Design patterns can improve the quality of the information models, so that the structure of the information model is more reasonable. Analyzing the specific business problems and using design patterns properly is an effective means in the model design.

\section{ACKNOWLEDGEMENTS}

This work was financially supported by the 985 Program of Minzu University of China (No. 10301-01404016).

\section{REFERENCES}

[1] Operations Support Systems(OSSs), http://www.hit.bme.hu/ jak ab/edu /litr/ TMN/oss.pdf

[2] Tabrizipoor,Oreimi,Pirhad. Investigation of Basic Services Interoperability Problem in Next Generation Network: Fourth European Conference on Universal Multiservice Network.Toulouse.France:346354,2007.

[3] St-Arnauld,Chabot. Integrated life-cycle power asset management for telecom operators. 32nd Telecommunications Energy Conference: Orlando,pp.1-7, 2010.

[4] OSS Transformation, http://www.osstransformation.com/What-is-OSSTrans formation.

[5] IETF RFC 1213, Management information base for network management of TCP/IP-based internets:MIB-II.1991.

[6] Distributed Management Task Force DSP0004,Common information model (CIM) Infrastructure version 2.7.0.2012. 
[7] ITU-T Recommendation M3100,Generic network information model,2000.

[8] TM Forum GB922 0-P.Information framework primer,2011.

[9] TM Forum, Connection and service management information model information agreement," June 2001.

[10] TM Forum, TMF608-MultiTechnology network management information agreement," August 2003.

[11] 3GPP TS 32.150, Integration reference point concept and definitions,2013.

[12] Yemini, Y. The OSI network management model. Communications Magazine, 31(5).20-29,1993.

[13] ITU-T Recommendation X.722, Information technology-open systems interconnection - structure of management information: guidelines for the definition of managed objects, 2000.

[14] ITU-T Recommendation M.3020. Management interface specification methodology,2011.

[15] TM Forum(Ed: Joel Fleck), The NGOSS lifecycle and methodology, GB927 , Ed. NGOSS R6.0, Document Version 4.5, November 2004.

[16] John Strassner, James Won-Ki Hong, and Kyo Kang. A framework for modelling and reasoning about network management resources and service to support information reuse. Information Reuse \& Integration. Las Vegas, NV:85-90,2009.

[17] Z.P. Gao ,L.M. Meng, X.S. Qiu. The interoperability and shared management information model. First Asia International Conference on Modelling \& Simulation. Phuket ,Thailand116-122,2007.

[18] Kenichi Ogaki, Masanori Miyazawa and Michiaki Hayashi. Integrating heterogeneous IT/network management models using linked data.2013 IFIP/IEEE International Symposium on Integrated Network Management. Ghent, Belgium:768-771,2013.

[19] Sethi, Anand, Gangopadhyay, Reddy, Gupta. An open framework for federating integrated management model of distributed it environment. Network Operations and Management Symposium. Salvador,Bahia:803$806,2008$.

[20] Z.P. Gao, X.S. Qiu, L.L. Rui. A novel modelling methodology of management information based on SID. Journal of Beijing University of Posts and Telecommunications,Vol.29 Sup:112-116,2006.

[21] Kisang Ok, Daniel Wonkyu Hong and Byungdeok Chung. Design methodology of operations supporting systems based on TMForum NGOSS. Global Telecommunications Conference: 1-5,2008. 